\title{
Frontières
}

LAMBIN, Michèle, Aider à prévenir le suicide chez les jeunes. Un livre pour les parents, Montréal, Hôpital Sainte-Justine, 2004, 262 p.

\section{Francine Gratton}

Volume 18, numéro 1, automne 2005

URI : https://id.erudit.org/iderudit/1074327ar

DOI : https://doi.org/10.7202/1074327ar

Aller au sommaire du numéro

Éditeur(s)

Université du Québec à Montréal

ISSN

1180-3479 (imprimé)

1916-0976 (numérique)

Découvrir la revue

Citer ce compte rendu

Gratton, F. (2005). Compte rendu de [LAMBIN, Michèle, Aider à prévenir le suicide chez les jeunes. Un livre pour les parents, Montréal, Hôpital

Sainte-Justine, 2004, 262 p.] Frontières, 18(1), 77-79.

https://doi.org/10.7202/1074327ar

Ce document est protégé par la loi sur le droit d'auteur. L'utilisation des services d'Érudit (y compris la reproduction) est assujettie à sa politique d'utilisation que vous pouvez consulter en ligne.

https://apropos.erudit.org/fr/usagers/politique-dutilisation/
Cet article est diffusé et préservé par Érudit.

Érudit est un consortium interuniversitaire sans but lucratif composé de l’Université de Montréal, l'Université Laval et l'Université du Québec à Montréal. Il a pour mission la promotion et la valorisation de la recherche. https://www.erudit.org/fr/ 
ouvrage de vulgarisation d'abord conçu pour les parents. L'auteure choisit d'ailleurs de s'adresser directement à eux dans un langage, le plus souvent, clair et simple tout en ayant le souci de définir au fur et à mesure des mots et expressions clés dans le domaine du suicide et des problèmes ou troubles de santé mentale. Fréquemment, on retrouve des descriptions, suggestions, recommandations sous forme de listes et quelques tableaux fort utiles sont insérés. En lisant les remerciements de l'auteure, les parents constateront avec plaisir que Mme Lambin, en plus de ses compétences en travail social et de ses vingt-cinq années d'expérience clinique auprès des jeunes et de leur famille, est aussi parent de cinq enfants. L'auteure divise son ouvrage en quatre sections. Après avoir traité de l'état des connaissances sur le suicide et de ses particularités, elle fait part des divers facteurs pouvant entraîner un état suicidaire chez des jeunes ainsi que des interventions possibles pour prévenir leur suicide et termine son exposé en abordant le rôle des parents. Elle prévient le lecteur qu'il pourra y avoir répétitions des éléments majeurs d'un chapitre à l'autre car le livre est conçu pour que les quinze chapitres puissent chacun être consultés "indépendamment les uns des autres" (p. 22). L'idée est excellente pour des parents qui, souvent, cumulent de multiples activités et manquent de temps. Ils peuvent ainsi avoir accès rapidement, et selon leurs besoins, aux informations très pertinentes contenues dans de courts chapitres, comprenant de 6 à 21 pages de texte très aéré. Chacun d'eux, sauf le premier, se termine par un résumé de quelques lignes et tout au long du texte, l'auteure encadre les éléments les plus importants. Cependant, la lecture de l'ouvrage intégral dans un laps de temps limité rend ces répétitions lourdes et donne une impression de déjà lu qui pourrait malheureusement inciter quelqu'un à en abandonner prématurément la lecture. On peut se demander si des redondances auraient pu être évitées en insérant, par exemple, un lexique à la fin.

La première partie, intitulée "Ce que l'on sait sur le suicide et ses particularités", inclut trois chapitres dans lesquels l'auteure traite des "principales croyances sur le suicide», des "indices de comportements suicidaires chez les jeunes" et de leur "conception de la mort». Le chapitre premier concernant les croyances débute par une invitation aux parents à répondre vrai ou faux à un mini-test de quinze questions. Comme l'auteure donne ensuite la bonne réponse tout en la justifiant par des explications précieuses sur le suicide et sur des problèmes de santé mentale qui y sont souvent liés, les parents vérifient l'état de leurs connaissances tout en prenant conscience de certains mythes. À la fin de ce chapitre, les parents savent, par exemple, que le suicide est une des principales causes de mort chez les jeunes, surtout chez les hommes de moins de 40 ans, qu'interviennent des aspects biopsychosociaux, que les familles de jeunes suicidaires peuvent être très normales et que l'influence du facteur héréditaire en tant que tel n'est pas démontré, qu'un jeune peut vivre un conflit de loyauté si un camarade lui confie ses intentions de suicide, etc. Le chapitre deux, intitulé "les indices de comportements suicidaires ", prend la forme de questions et d'inquiétudes de parents concernant l'adolescence en lien avec des indices de préoccupations suicidaires à cette étape de vie. Après avoir défini ce qu'on entend par signes précurseurs, l'auteure traite brièvement de points culminants à l'adolescence tout en remettant en question l'élément de crise d'adolescence qu'on y associe trop souvent. Après avoir traité de la crise suicidaire en termes $d^{\prime}$ 'impasse relationnelle» (p. 47) [expression un peu compliquée utilisée par l'auteure à quelques reprises], tout en étant rassurante et en proposant des façons d'être aux parents, elle les incite à détecter les expressions de souffrance et de désarroi chez leurs jeunes et à être attentifs à tout changement. À l'aide d'exemples très bien choisis d'un adolescent et d'une adolescente suicidaires, elle explique avec nuance ces quatre types $d$ 'indices suicidaires: les messages verbaux directs et indirects, les indices affectifs et les indices comportementaux. La structure du chapitre trois ressemble à celle du chapitre précédent et traite non seulement de la "conception de la mort" qu'ont les jeunes mais plus spécifiquement de celle de la mort par suicide. La compréhension qu'ont les enfants des notions de mort et de suicide y est expliquée et elle est très bien illustrée par l'histoire de Sandrine (8 ans). Sans être alarmiste, l'auteure souligne aussi l'influence dangereuse des émotions fortes, typiques à l'adolescence, sur les états suicidaires des jeunes. Les réactions possibles des parents face à leur enfant suicidaire ainsi que des suggestions d'attitudes y sont aussi discutées. 
La deuxième partie du livre, intitulée «Ce qui peut entraîner l'état suicidaire chez un jeune ", comprend cinq chapitres qui traitent de trois sujets: les facteurs psychosociaux; les vulnérabilités individuelles; les facteurs précipitants. Le chapitre quatre concernant "les facteurs psychosociaux" traite brièvement de l'interaction entre l'environnement et les conduites suicidaires des jeunes. Le titre est plus ou moins représentatif du contenu en ce sens que l'auteure limite son propos à l'influence de la famille sur le bien-être du jeune et ne traite aucunement de l'école, milieu fréquenté quotidiennement plusieurs heures par les garçons et les filles à l'adolescence. Après avoir souligné la nécessité pour un jeune de trouver un sens à sa vie, l'auteure insiste sur l'influence d'un milieu de vie comme la famille sur son épanouissement personnel et le cheminement d'un jeune vers l'autonomie ou encore sur la mise en place d'un état suicidaire. Bien que nuancés, très pertinents et susceptibles de favoriser la réflexion des parents, certains propos de l'auteure, qui précise bien ne pas vouloir idéaliser la famille, prennent, malgré tout, l'aspect de recettes de bonheur familial (p. 71). Mais l'énumération des caractéristiques de la "famille saine» (p. 71) propose aussi des points de repère au sujet du climat à favoriser dans la famille. Les chapitres cinq à huit inclusivement sont consacrés aux «vulnérabilités individuelles», incluant les problèmes de santé mentale et les traits de personnalité, susceptibles d'entraîner aussi un état suicidaire. Avant d'entreprendre la synthèse de huit problèmes de santé mentale très bien choisis, l'auteure utilise le chapitre cinq, intitulé "Les vulnérabilités individuelles: les problèmes de santé mentale», pour introduire le concept de problèmes de santé mentale. Après avoir insisté sur les mythes entourant ces problèmes et la nécessité de les traiter au même titre que les problèmes de santé physique, elle définit ce concept, fait un survol des causes possibles, donne un aperçu des troubles mentaux qu'elle décrira dans les chapitres subséquents tels qu'ils le sont dans le DSM-IV et rédige une liste, bien éclairante pour des parents, des principaux symptômes. Tout comme elle le fera dans chacun des chapitres ultérieurs, elle souligne l'importance du soutien de la famille et du réseau du jeune tout comme la nécessité de consulter rapidement un professionnel de la santé. Par contre, peut-être pour ne pas décourager les parents, elle ne fait aucunement mention des difficultés possibles d'accès aux services de certains professionnels, comme les pédopsychiatres. D'ailleurs, elle prend soin de rédiger une liste appropriée de ressources de toutes sortes à la fin de son ouvrage.

Le chapitre six traite de "La dépression" et débute par des statistiques reliant dépression et conduites suicidaires. L'auteur définit ensuite ce problème tout en abordant les multiples causes possibles, dont les facteurs familiaux, et en prenant soin de distinguer déprime et dépression majeure. Après avoir averti les parents de la spécificité des symptômes pour chaque jeune, elle en dresse une liste regroupée de la sorte: les manifestations visibles, les symptômes physiques, les symptômes subjectifs et les changements de comportements. Le tout est synthétisé dans un tableau (p.101) et bon nombre de ses propos, qu'elle veut rassurants et non alarmistes, sont illustrés par des témoignages de parents, d'adolescents, de jeunes qui ont tenté de se suicider et d'autres qui ont mis fin à leurs jours. Le chapitre sept est consacré aux "autres problèmes de santé mentale ».

Malgré des définitions précises contenus dans le chapitre cinq, il y a confusion de certains termes; le chapitre aurait pu être intitulé "les autres troubles mentaux» étant donné qu'on y traite de "maladies psychiatriques selon les critères du DSM-IV» (p. 79). L'auteur y présente la synthèse d'un choix judicieux de sept troubles mentaux: les troubles de l'humeur (dysthymie, dépression saisonnière, trouble bipolaire); les troubles anxieux (phobie sociale, troubles d'anxiété généralisée, trouble obsessionnel compulsif, trouble panique, état de stress post-traumatique); les troubles de dépendance ou d'abus de drogues ou d'alcool; les troubles de la conduite incluant les troubles oppositionnels; le déficit de l'attention et I'hyperactivité; les psychoses (trouble psychotique, psychose toxique, schizophrénie); et les troubles alimentaires (anorexie, boulimie). En lisant ce chapitre, des parents peuvent acquérir des connaissances fort utiles car chacun de ces troubles est systématiquement défini dans un langage compréhensible; des distinctions sont établies par rapport à d'autres troubles présentant des éléments communs; la symptomatologie est décrite simplement en termes de comportements souvent observables; les risques de suicide, à des moments cruciaux, sont discutés tout en demeurant rassurant pour des parents; des facteurs de protection, susceptibles d'influencer une attitude aidante de la part de la famille, sont mentionnés et de précieux conseils aux parents sont mis en évidence dans un encadré (p.129). Le chapitre huit clôt la section des vulnérabilités individuelles en présentant "les traits de personnalité ", aussi appelés tempérament, susceptibles d'influencer un état suicidaire. L'auteure construit son propos, sous forme de nombreuses énumérations, autour des prédispositions et des obstructions au bonheur. Elle énumère des qualités comme l'optimisme, l'ouverture d'esprit, les habiletés relationnelles, la persévérance dans l'effort, la générosité du cœur, etc., qui facilitent la capacité d'être heureux, de vivre avec les autres et de surmonter les difficultés. Par la suite, elle démontre avec justesse comment d'autres traits de personnalité comme l'impulsivité, le perfectionnisme, le pessimisme, une faible tolérance à la frustration, un besoin insatiable d'être aimé, I'anxiété, la culpabilité, etc., teintent les expériences d'un jeune au point de le prédisposer à un état suicidaire.

Le chapitre neuf, dernier de cette deuxième partie consacrée à ce qui peut entraîner un état suicidaire, traite des "facteurs précipitants" que représentent ces «événements qui se déroulent dans la vie d'un jeune et qui, par leur influence à ce moment précis de la vie, peuvent entraîner un geste suicidaire» (p. 139). Tout en précisant que ce qui importe n'est pas tant l'événement en soi mais ce qu'il représente pour un jeune, I'auteur explique comment ces événements peuvent devenir la goutte d'eau qui fait déborder le vase: le décès d'un proche, une rupture amoureuse, des relations familiales conflictuelles, l'isolement social et la difficulté à développer un sentiment d'appartenance à un groupe de pairs, le harcèlement et l'intimidation, des événements traumatiques, des problèmes légaux et des conflits avec l'autorité, des problèmes scolaires, l'abus de drogues et d'alcool, les dates symboliques et, enfin, les nuits blanches. À cause de la probabilité que certains de ces événements soient vécus par leur jeune, la lecture de ce chapitre est particulièrement enrichissante pour des parents ainsi que le témoignage d'un jeune qui rend compte de l'influence, sur sa tentative de suicide, des difficultés vécues à l'école. Bien que quelques références soient notées dans le texte, il aurait été avantageux pour un lecteur intéressé à approfondir un sujet en particulier (telles les «nuits blanches», notion peu traitée dans les écrits scientifiques) de connaître précisément les sources bibliographiques de l'auteure.

Quatre chapitres constituent la troisième partie de ce livre, intitulé «En intervenant». Le chapitre dix, "Le suicide: facteurs de risque», souligne des facteurs susceptibles de provoquer une situation de crise chez un jeune. L'auteure est rassurante pour les parents en précisant que l'accumulation des facteurs mentionnés dans ces pages ne rendra pas nécessairement un jeune suicidaire. Bien que plusieurs éléments aient déjà été vus dans des chapitres antérieurs, comme la présence de troubles psychiatriques associée à $90 \%$ des suicides complétés (résultats d'autopsie psychologiques), l'impulsivité, le décès d'un proche, etc., on y insère quelques éléments nouveaux comme l'impact des médias, les notions d'intentionnalité et de létalité (qu'on définit très bien) du geste suicidaire, l'orientation sexuelle, l'accessibilité des moyens. Bien que cet ouvrage en soit un de vulgarisation, il aurait été utile de préciser, de varier et de présenter de façon plus critique certaines sources de référence afin de rendre plus crédibles certaines affirmations et pour justifier davantage certains propos qu'on pourrait remettre en question (tel le modèle de l'entonnoir à la page 156). Pour ne donner qu'un exemple, le nombre de jeunes (surtout d'adolescents ou $d^{\prime}$ adolescentes qui ont entre 12 et 16 ans) qui cumulent, à la fois et en même temps, une dépression, des problèmes de conduite et un (véritable) abus de drogue (p. 159) peut être heureusement très limité (hormis ceux qui consultent dans des milieux aussi spécifiques que les centres jeunesse). Dans une note de bas de page (p. 165), on sent aussi le malaise de l'auteure à appuyer une affirmation sur les résultats d'une étude qualitative. On peut difficilement le lui reprocher car plusieurs chercheurs ont malheureusement aussi cette attitude. Mais ce genre de situations est si fréquent qu'il s'impose ici de rappeler que les résultats d'une étude qualitative, comme celle de Dorais, peuvent être aussi fiables que ceux d'une étude quantitative dont l'échantillon serait composé de centaines de sujets. Des critères de scientificité très précis existent pour juger de la qualité de ces études et ils ne se limitent surtout pas au 
nombre de sujets. D'ailleurs, si on consultait davantage les recherches qualitatives dans le domaine du suicide, on aurait accès, par exemple, à des informations très riches concernant l'enchaînement (plus ou moins rapide) des événements lors d'un processus suicidaire (p.157).

Le chapitre onze est consacré aux "facteurs de protection" qui, lorsque présents, diminuent les risques qu'un jeune adopte une conduite suicidaire lorsqu'il est aux prises avec des problèmes. L'auteur reprend des éléments déjà mentionnés concernant les jeunes comme ses capacités à aller chercher de l'aide, ses compétences relationnelles, etc. D'autres facteurs très appropriés sont aussi élaborés tels que les valeurs spirituelles, I'influence du genre sur la demande d'aide (ces propos auraient pu être appuyés par des écrits de Germain Dulac) et la qualité de la prise en charge thérapeutique du jeune. $\mathrm{Ce}$ dernier élément, qui sera repris plus longuement dans le chapitre suivant, est précieux pour des parents qui, pour diverses raisons, peuvent vivre difficilement et avec anxiété le moment où ils confient leur jeune à des intervenants. L'auteure les informe des objectifs généralement poursuivis par les intervenants et des avantages pour les jeunes en difficulté d'être suivis en thérapie individuelle ou de groupe tout en bénéficiant du soutien constant de leurs parents et de leurs camarades. Le chapitre douze permet l'acquisition de connaissances théoriques qui aiderait le lecteur à examiner les exemples qui seront proposés dans le chapitre suivant, au sujet de I'«intervention auprès d'un jeune suicidaire». Après avoir souligné l'importance que des parents demandent de l'aide à la moindre inquiétude concernant leur jeune, l'auteure les informe de la possibilité de bénéficier d'interventions de première ligne accessibles dans la communauté (milieux scolaires, CLSC, cliniques médicales, etc.) et elle indique si les services requis doivent être spécialisés, d'interventions de deuxième ligne (centres jeunesse, cliniques externes de pédopsychiatrie, etc.). Tout en soulignant la rareté de cette situation (on aurait pu se référer aux travaux de J.-J. Breton), elle décrit l'attitude "idéale» du jeune qui fait part de sa souffrance à un intervenant et demande de l'aide. Sont ensuite proposées des pistes d'intervention basées sur la concertation des intervenants qui doivent tenir compte de leurs limites, faire preuve de respect ainsi que de loyauté face aux jeunes et transmettre de l'espoir tout en établissant un pacte de non-suicide avec lui. Après avoir effleuré le travail fait dans les écoles, des stratégies sont proposées aux parents afin d'amener leur jeune en consultation, lorsque nécessaire, pour procéder d'abord à une évaluation de son état. L'auteure dresse la liste des objectifs poursuivis lors de cette première étape et énumère les objectifs inhérents à une prise en charge tout en faisant un survol des diverses approches thérapeutiques (cognitivo-comportementale, systémique structurelle et stratégique, etc.). L'auteur termine ce chapitre en présentant des informations très précieuses concernant la prise de la médication et, plus particulièrement, celle des antidépresseurs. Le chapitre treize intitulé "Aider Sandrine, Amélie et Carl-Éric » complète bien cette partie en invitant les parents, à l'aide des histoires de deux filles et d'un garçon, à intégrer leurs connaissances théoriques concernant l'intervention. Elle leur demande: que feraient-ils comme interventions dans une première situation? Comment aimeraient-ils qu'un entraîneur de basketball ou qu'un enseignant intervienne dans les deux autres situations?

La dernière partie du livre, intitulée "Le rôle des parents", comprend deux chapitres. Le chapitre quatorze donne une série de bons conseils aux parents d'un jeune suicidaire. Malgré la culpabilité, la tristesse et l'inquiétude qu'ils peuvent ressentir, l'auteure les invite à garder espoir et leur rappelle que leur jeune a grand besoin de leur amour, de leur écoute, etc. Elle les invite à partager leurs craintes avec leur enfant, à obtenir l'aide des intervenants, à s'impliquer activement dans tous les aspects de son traitement, à éviter toute banalisation du suicide tout en continuant à prendre soin de leurs autres enfants. Le chapitre quinze "énumère" des "attitudes parentales préventives» et se clôt par un riche témoignage d'un jeune de dix-neuf ans. L'auteure fait la liste des nombreuses exigences du rôle de parents. Elle rappelle leurs responsabilités quant à la qualité de la vie familiale et l'épanouissement de chacun des membres. Après avoir souligné que chaque enfant est unique, elle les invite, par exemple, à aider leur enfant: à augmenter son seuil de tolérance à la frustration, à développer des habiletés relationnelles, à croire en sa capacité à surmonter des peines de la vie, etc. Ces sages conseils (on ne peut certainement pas "être contre la vertu») qui comportent toutefois de grandes exigences pour les parents et sur lesquelles on s'attarde peu, se poursuivent aussi dans la conclusion. On ne peut s'empêcher de penser que l'application de "ce qu'il faut faire» peut être bien compliquée considérant les difficultés de toutes sortes que vivent des parents. Mais enfin...

Bref, malgré quelques limites mentionnées tout au long du texte, ce livre est une lecture à recommander chaleureusement à des parents préoccupés par le phénomène du suicide chez les jeunes, mais aussi à bien d'autres personnes qui pourront bénéficier non seulement de la synthèse des connaissances qu'on y retrouve, mais de l'expérience de madame Lambin, tant comme clinicienne que comme parent, car celle-ci transparaît tout au long du texte.

Francine Gratton 